Samples of serum and cerebrospinal fluid obtained before, during, and after the period of treatment with adrenocorticotrophic hormone and plasmapheresis were assayed for complement fixing antibrain antibodies ${ }^{1}$ and found to react with homogenate of human white matter $(1: 30 \mathrm{wt} / \mathrm{vol}$ in veronal buffer). The IgG class of the antibodies was shown by absorption and elution on Protein A-Sepharose CL-4B (Pharmacia, Sweden). Before plasmapheresis the IgG index was 0.74 and the cerebrospinal fluid antibody index ${ }^{5}$ for antibrain antibodies was $27 \cdot 4$ (upper normal limit $=2$ ). Whereas treatment with adrenocorticotrophic hormone did not seem to affect the serum level of antibrain antibodies, plasmapheresis caused them to be virtually eliminated from serum concomitant with a pronounced drop in
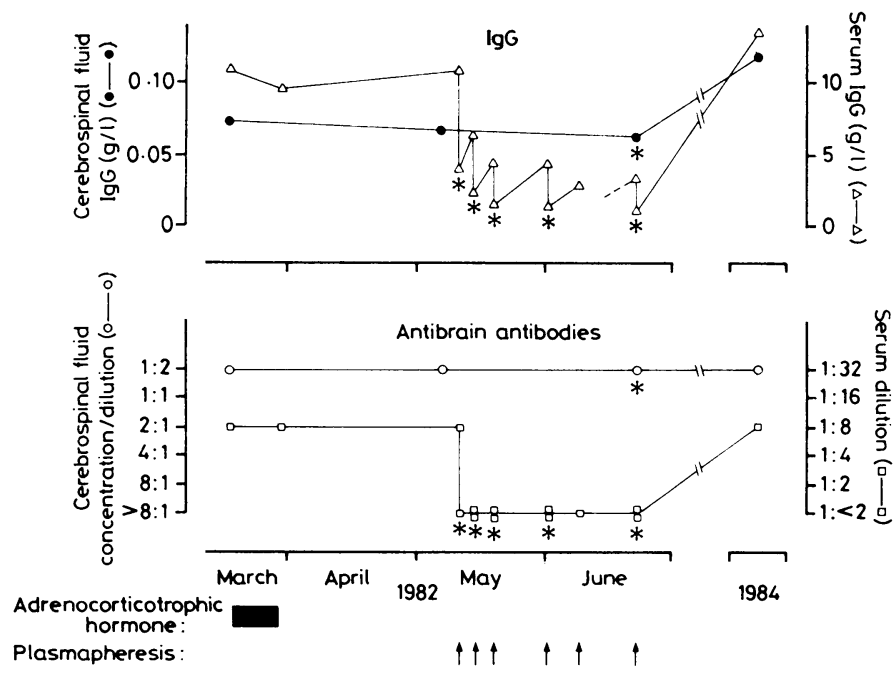

IgG concentrations and antibrain antibodies before, during, and after plasmapheresis. Before titration of antibodies cerebrospinal fluid was concentrated and rediluted to give multiples of original IgG concentration.

^Samples obtained within 12 hours after plasmapheresis. serum IgG concentrations. The antibody titre and IgG concentration in cerebrospinal fluid, however, remained unchanged (figure). Cerebrospinal fluid obtained just after the last session of plasmapheresis showed the same oligoclonal IgG pattern as cerebrospinal fluid obtained shortly before treatment.

\section{Comment}

The important finding in this study was the persistence during plasmapheresis of antibrain antibodies in cerebrospinal fluid along with an essentially unchanged IgG concentration and oligoclonal IgG pattern in this fluid. These observations are consistent with intrathecal production of both IgG and antibrain antibodies. This conclusion is supported by the high IgG and antibody indexes.

Thus the therapeutic failure of plasmapheresis in this and other patients with multiple sclerosis may be related to the inability of this method to remove antibrain antibodies, and perhaps other humoral factors, from the compartment where they probably react with the central nervous system-that is, the intrathecal space. The patients with multiple sclerosis most likely to profit from plasmapheresis may be those with a predominantly extrathecal distribution of such factors.

\section{References}

1 Ryberg B. Antibrain antibodies in multiple sclerosis. Relation to clinical variables. $\mathcal{F} \mathrm{Neurol} \mathrm{Sci}$ 1982;54:239-61.

2 Stefoski D, Schauf CL, McLeod BC, Haywood CP, Davis FA. Plasmapheresis decreases neuroelectric blocking activity in multiple sclerosis. Neurology 1982;32:904-7.

3 Caparelli R, Inzitari D, Sita D, et al. Plasmapheresis in multiple sclerosis: cerebrospinal fluid changes after treatment. In: Gonsette RE, Delmotte P, eds. Immunological and clinical aspects of changes after treatment. In: Gonsette RE, Delmotte
multiple sclerosis. Lancaster: MTP Press, 1984:57-60.

multiple sclerosis. Lancaster: MTP Press, 1984:57-60.
4 Schumak KH, Rock GA. Therapeutic plasma exchange. N Engl f Med 1984;310:762-71

4 Schumak KH, Rock GA. Therapeutic plasma exchange. N Engl f Med 1984;310:762-71.
5 Ryberg B. Extra- and intrathecal production of antinerve and antibrain antibodies in Guillain-Barre Ryberg B. Extra-and intrathecal production of antinerve and antibrain antibod
syndrome: evaluation by an antibody index. Neurology 1984;34:1378-81.

(Accepted 17 fuly 1985

\title{
Are coronary artery spasm and progressive damage to the heart associated with the hyperventilation syndrome?
}

\author{
LEISA J FREEMAN, P G F NIXON
}

\begin{abstract}
A case of coronary artery vasospasm was studied in a man with a four year history of angina. He had evidence of symptomatic hyperventilation during a spontaneous episode of chest pain. When asked to hyperventilate the pain in his chest and ST elevation were reproduced in the same leads as occurred during the spontaneous attack. This may be the first reported case of spontaneous hyperventilation producing vasoconstriction, and the patient's previous admissions to the coronary care unit may have been associated with coronary vasospasm induced by hyperventilation.

When patients with variant angina report pains in the chest in association with dizziness and breathlessness hyperventilation
\end{abstract}

Cardiac Department, Charing Cross Hospital, London W6 8RF

LEISA J FREEMAN, MRCP, registrar

PG F NIXON, FRCP, consultant cardiologist

Correspondence to: Dr Freeman should be considered to be a possible cause of the symptoms. As coronary vasospasm is increasingly implicated in angina after myocardial infarction the role of hyperventilation should be considered more often.

\section{Introduction}

Coronary vasospasm, either spontaneous or provoked at catheterisation, has been confirmed as the pathogenic mechanism in variant angina.' We describe a patient with coronary artery vasospasm and suggest an aetiological role for the associated hyperventilation.

\section{Case report}

A 62 year old foreman was admitted for assessment of chest pain. He had a four year history of exertional, nocturnal, and rest angina. Breathlessness and giddiness were associated with the pain. He had had 14 admissions to a local coronary care unit with suspected myocardial infarctions without changes in enzyme concentrations to confirm the diagnosis. ST elevation was noted on at least six occasions. On the first eight admissions the electro- 
cardiogram had initially shown a left bundle branch block that resolved with relief of pain. After 1981 the left bundle branch block persisted. He had a normal coronary arteriogram in 1980 . Before admission he had been treated with propranolol, nifedipine, and isosorbide dinitrate. Examination showed a prominent fourth heart sound and an irregular respiratory pattern with frequent sighs.

On the night of admission he had a spontaneous attack of severe chest pain associated with breathlessness. The electrocardiogram showed acute ST elevation in $V_{2}$ and $V_{3}$. Blood gas analysis showed hypocarbia $(\mathrm{pH}=7 \cdot 6$, arterial oxygen pressure $=112 \mathrm{~mm} \mathrm{Hg}$, arterial carbon dioxide pressure $=12$
The exact aetiology of coronary arterial spasm is still unclear, but Maseri and Chiercha suggested that it may represent local hype sensitivity to a normal physiological stimulus at certain times. ${ }^{4}$. have proposed that spontaneous hyperventilation may be a trigger and the associated increased sympathetic nervous activity and raised catecholamine concentrations may sensitise the coronary bed.'

When patients with variant angina report pains in the chest association with dizziness and breathlessness, expressed as am inability to take a deep and satisfying breath or a need for frequegt

$\begin{array}{llllll}\text { ST segment elevation } & V_{2} & 3.6 & 5.3 & 5.7 & 3.8 \\ \text { in } \mathrm{mm}, 80 \mathrm{~ms} \text { from } & V_{3} & 4.1 & 4.7 & 6.4 & 3.7 \\ \text { the J point } & & & & \\ \begin{array}{l}\text { End tidal carbon } \\ \text { dioxide tension }\end{array} & 37.5 & 19.4 & 16.2 & 24.7\end{array}$
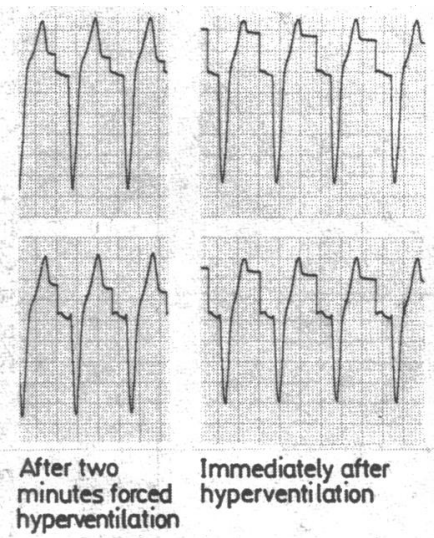
Immediately after
hyperventilation At rest
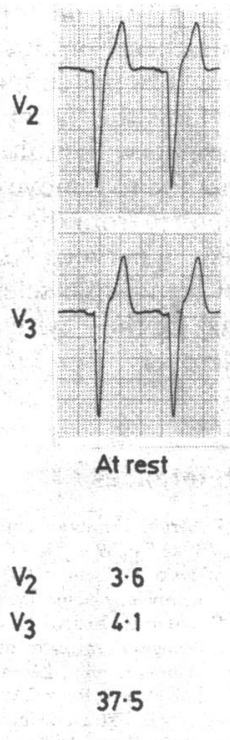

Hyperventilation provocation test (forced hyperventilation for three minutes at 60 breaths/minute).

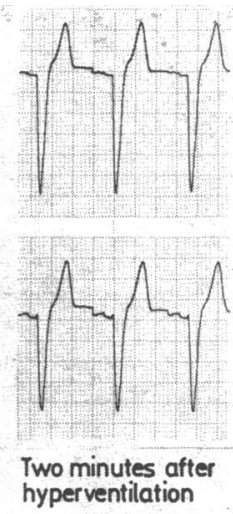

$\mathrm{mm} \mathrm{Hg}$ ). The ST segment returned to normal after relief of pain with pethidine. A normal exercise test to 140 beats/minute was performed later when the patient was asymptomatic (end tidal carbon dioxide tension $=38 \cdot 7 \mathrm{~mm} \mathrm{Hg}$ )

A forced hyperventilation test reproduced ST segment elevation in $\mathrm{V}_{2}$ and $V_{3}$ (figure). Chest pain occurred after 2.5 minutes of hyperventilation and persisted for a further 1.5 minutes after hyperventilation was stopped. A second coronary arteriogram in 1982 showed minimal stenosis of the circumflex artery and slight left ventricular enlargement.

The cause of his symptoms was explained to him. He was taught diaphragmatic breathing control, ${ }^{2}$ particularly for use during times of anxiety and emotion, which had previously precipitated his pain. $\mathrm{He}$ remained free from symptoms at nine months.

\section{Discussion}

This patient had evidence of symptomatic hyperventilation, confirmed by the arterial blood gas finding of profound hypocapnia during a spontaneous episode of chest pain with ST segment elevation. When he was asked to hyperventilate it was possible to reproduce chest pain and ST elevation in the same leads as occurred during the spontaneous attack.

Hyperventilation has been used during angiography to provoke coronary artery spasm with ST elevation and pain. ${ }^{3}$ Although hyperventilation induced ST elevation and pain occurred earlier in our case than in those patients previously described, ${ }^{3}$ the time sequence was similar. The faster rate of hyperventilation used during our provocation test may explain this finding. We believe that this is the first reported case of spontaneous hyperventilation producing vasoconstriction.

We further speculate that his regular admissions may have been associated with coronary vasospasm induced by hyperventilation. Ischaemia from such repeated episodes of spasm may have been responsible for the development of the left bundle branch block and left ventricular enlargement. sighing, hyperventilation should be considered to be a possibte cause of the symptoms. Provocation tests (either hyperventilation of exercise), which include concurrent monitoring of both end tid? tant information in these patients. As coronary vasospasm $\frac{0}{85}$ increasingly implicated not only in unstable angina but also angina after myocardial infarction we suggest that the role hyperventilation should be considered more often.

\section{References}

Oliva PB, Potts DE, Pluss RG. Coronary artery spasm in Prinzmetal angina documentation.

coronary angiography. $N$ Engl f Med 1973;288:245-51.
2 Lum CL. Breathing exercises in the treatment of hyperventilation and chronic anxiety states. The Chest, Heart and Stroke foumal 1977;2:7-11.

3 Girotti LA, Crosatto JR, Messutti H, et al. The hyperventilation test as a method for developi@ successful therapy in Prinzmetal angina. Am f Cardiol 1982;49:834-41.

4 Maseri A, Chiercha S. Coronary artery spasm, definition, diagnosis and consequences. Prof Cardiovasc Dis 1982;25:169-92.

5 Freeman LJ, Nixon PGF. Chest pain and the hyperventilation syndrome-some aetiologi considerations. Postgrad Med $\mathcal{J}$ (in press).

\section{YEARS AGO} disappointed in love, jumped from the Suspension Bridge at Clifton (t) highest bridge in England) into the bed of the river Avon, and escaped with very slight injury. Her case, which is of surgical interest, has in this psychological bearing, for we are informed that, before she left the infirmary, she received three offers of marriage. (British Medical Foumat 1885;i:1210.) carbon dioxide tension and electrocardiograms, may yield impom

A curious incident is noted in respect to the young woman who, beim 